\title{
Reduction in the therapeutic intensity of abortive migraine drug use during ACE inhibition therapy-a pilot study ${ }^{\dagger}$
}

\author{
H. Rahimtoola $\mathrm{PhD}^{1,2 *}$, H. Buurma ${ }^{2}$, C. C. Tijssen ${ }^{3}$, H. G. Leufkens ${ }^{1}$ and A. C. G. Egberts ${ }^{1,4}$ \\ ${ }^{1}$ Department of Pharmacoepidemiology and Pharmacotherapy, Utrecht Institute for Pharmaceutical Sciences (UIPS), \\ Utrecht, The Netherlands \\ ${ }^{2}$ SIR Institute for Pharmacy Practice Research, Leiden, The Netherlands \\ ${ }^{3}$ Department of Neurology, St. Elisabeth Hospital, Tilburg, The Netherlands \\ ${ }^{4}$ Hospital Pharmacy Midden Brabant, Twee Steden Hospital and St. Elisabeth Hospital, Tilburg, The Netherlands
}

\section{SUMMARY}

Introduction Since a few case reports have demonstrated some beneficial effects of angiotensin converting enzyme (ACE) inhibitors in migraine prevention, we were interested in studying the impact of ACE inhibitors and angiotensin II receptor antagonists (Ang II) on the consumption of specific abortive migraine drugs and, therefore, indirectly on the frequency of migraine attacks.

Methods Data from a large prescription database involving 95 patients initiating a specific abortive migraine drug (ergotamine or a triptan) and subsequently treated with either an ACE inhibitor or angiotensin receptor antagonist (index group: ACE/Ang II) or diuretic (reference group) were analysed. The effects of ACE/Ang II inhibition as well as diuretic therapy on reducing the frequency of migraine attacks were assessed by measuring the mean consumption of abortive migraine drug use, in DDDs per month ('therapeutic intensity'), before, during and after ACE/Ang II or diuretic therapy. A 'therapeutic fluctuation intensity estimate' of abortive migraine drug use for all patients was likewise calculated.

Results On an individual level, the therapeutic intensity (TI) fluctuation estimate, 'during' relative to 'before' ACE diuretic therapy, was significantly larger for the ACE/Ang II group (62\% reduction) than for the diuretic group (24\% reduction) $(p=0.02)$. For patients who continued abortive migraine drug use during and after ACE/Ang II or diuretic therapy, a significantly larger reduction in this estimate was observed during ACE/Ang II inhibition (68.9\%) compared to during diuretic therapy ( $10.5 \%$ increase) $(p=0.004)$. The TI fluctuation estimate, after relative to 'during', had increased by $50.3 \%$ after ACE/Ang II inhibition and had reduced by $22.2 \%$ after diuretic treatment $(p=0.1)$.

Conclusions A clear reduction in the TI of abortive migraine drug use during the use of ACE inhibitors as compared to diuretic treatment was observed. Our findings may indirectly support a positive effect of ACE/Ang II inhibition on the frequency and severity of migraine attacks, as observed in other studies and reports. Copyright (C) 2003 John Wiley \& Sons, Ltd.

KEY WORDS - migraine; ACE inhibitors; triptans

\footnotetext{
*Correspondence to: Dr H. Rahimtoola, Department of Pharmacoepidemiology and Pharmacotherapy, Utrecht Institute for Pharmaceutical Sciences (UIPS), Sorbonnelaan 16, PO Box 80082, 3508 TB Utrecht, The Netherlands. E-mail: h.rahimtoola@pharm.uu.nl ${ }^{\dagger}$ No conflict of interest was declared.

Contract/grant sponsors: The Royal Dutch Association for the Advancement of Pharmaceutical Sciences (KNMP), The Hague, The Netherlands; SIR Institute of Pharmacy Practice Research, Leiden, The Netherlands.
}

\section{INTRODUCTION}

Migraine is a common disabling condition that can significantly limit and impair the patient's health-related quality of life. ${ }^{1}$ Despite the recent advances in migraine abortive therapy, such as the availability of sumatriptan and the second generation triptans, a significant number of migraine patients are unable to achieve satisfactory results from specific or 
non-specific abortive migraine therapy. In these patients, the initiation of prophylactic medication can therefore be considered a therapeutic option. ${ }^{2}$ In clinical practice, this form of treatment is frequently unable to provide sustained benefit and is often limited due to a wide spectrum of side-effects. The use of $\beta$-blockers, serotonin agonists, calcium channel blockers, clonidine and valproic acid for migraine prophylaxis can reduce the frequency of migraine attacks by about $50 \%$ as compared to placebo. The few controlled comparative clinical trials conducted so far have failed to prove any superiority of one migraine prophylactic medication to another. This indicates the need to further clarify the pathogenesis of migraine and investigate other drugs that can enhance the therapeutic options of migraine prevention.

Some clinical trials and case reports have demonstrated beneficial effects of other drugs such as the selective serotonin reuptake inhibitors, high dose riboflavin, magnesium and even the coumarin derivatives in the prophylactic management of migraine. ${ }^{3-6}$ There is increasing evidence that use of angiotensin converting enzyme (ACE) inhibitors can substantially reduce the frequency and severity of migraine attacks. Quite recently, a controlled clinical trial demonstrated a clear improvement of migraine, in terms of headache duration and severity, during ACE inhibition therapy with lisinopril when compared to placebo. ${ }^{7}$ Although the underlying mechanism associated with the possible improvement of migraine due to ACE inhibition therapy has not been fully elucidated, it has been suggested that the effects of ACE inhibitors are related to their ability to enhance the effects of norepinephrine and serotonin on vascular tone. ${ }^{8}$

It has therefore, been suggested that the use of lisinopril and other ACE inhibitors including angiotensin II receptor antagonists (Ang II) should be considered a viable long-term therapeutic approach to migraine prevention. ${ }^{9,10}$ It has however, not been shown whether the positive effects on migraine are shared by all ACE I and II inhibitors. This means that the possible reduction in migraine attack frequency and severity due to the use of angiotensin receptor antagonists as a group requires further assesment.

The objective of this observational retrospective follow-up study was to evaluate the nature and strength of the association between angiotensin receptor antagonist therapy and severity of migraine by evaluating the intraindividual change in specific abortive migraine drug use before, during and after the initiation of ACE I or II inhibition therapy.

Copyright (C) 2003 John Wiley \& Sons, Ltd.

\section{METHODS}

\section{Study setting}

The study used prescription data from the PHARMORLS database located at the University of Utrecht, the Netherlands, covering the period 1985-1998. This database has been described in full elsewhere. ${ }^{11}$ In brief, the system was designed in 1985 to provide relevant demographic and prescription data at an individual level for five medium-sized cities in the Netherlands from 1985-1989. Since 1990 it has been further updated, covering a total of six cities $(n=300000)$, and from 1993 to 1998, eight cities $(n=450000)$. In view of a high patient-pharmacy registration commitment in the Netherlands, in addition to sophisticated pharmacy software currently available, the prescription medication information for each patient is virtually complete.

Each registered person is identified with an anonymous unique patient identification code which allows for the observation of patient medication use in time. Retrievable information per prescribed medicine includes date of dispensing, drug, dosage regimen, quantity supplied (defined daily doses) and type of prescriber. Patient information per prescribed medicine includes gender and date of birth. The database does not provide information concerning the indications of use of the medicines, in this case the diagnosis of migraine versus cluster headache, or complete registration of non-prescription medicines (e.g. overthe-counter (OTC) use of salicylates or paracetamol).

\section{Study population}

For this study, patients presenting more than one prescription for an abortive migraine drug (ergotamine, naratriptan, rizatriptan, sumatriptan and zolmitriptan) as well as a prescription for an angiotensin I or II antagonist ('ACE/Ang II inhibitors') for the first time from 1st January 1985 to 31st December 1998 were initially identified using the Anatomical Classification Codes (ATC) for each drug. ${ }^{12}$ As a reference group, patients using abortive migraine drugs as well as diuretic treatment (loop, potassium sparing or thiazide) were included.

For each patient included in the study, the date of first prescription of an abortive migraine drug was termed the 'start date analgesia'.

Patients identified above were subsequently categorised into two groups: patients commencing ACE inhibition therapy (index group, $n=165$ ) or patients commencing diuretic therapy (reference group, $n=229$ ) following the start date of analgesia. The first

Pharmacoepidemiology and Drug Safety, 2004; 13: 41-47 
Before

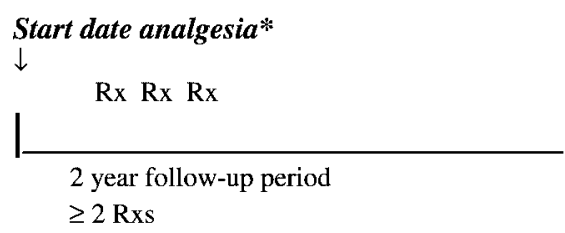

During Start date ACE or Diuretic $\downarrow$

After

Start date* corresponds to the date of first prescription of specific abortive migraine drug

Start date ACE or Diuretic ${ }^{\gamma}$ corresponds to the date of first prescription of either angiotensin receptor antagonist or diuretic treatment

$\mathbf{R x}$ corresponds to an abortive migraine drug prescription (ergotamine or triptan)

Figure 1. Study design

prescription of ACE inhibition or diuretic therapy was termed 'start date ACE or diuretic'.

For eligibility, patients had to fulfil all of the following criteria (Figure 1):

- duration of ACE and diuretic therapy of at least two years, defined as the time-span between the first and last prescription of the particular drug;

- not using an ACE inhibitor and diuretic simultaneously during evaluation period;

- not having commenced migraine prophylactic therapy (propranolol, metoprolol, pizotifen, clonidine, flunarizine, methysergide, verapamil, amitriptyline or valproic acid) during the entire study period; and

- the presentation of at least two prescriptions of the abortive migraine drug during a 2-year observation period preceding the 'start date ACE or diuretic'.

After having satisfied the eligibility criteria, 95 patients were included in the study population, of which $47(49 \%)$ and $48(51 \%)$ had been prescribed either an ACE inhibitor or diuretic respectively.

\section{Data analysis}

For each identified patient, a few baseline characteristics were determined including age (determined at start date analgesia), gender, type of abortive migraine drug use, start date ACE or diuretic and duration of ACE inhibition and diuretic therapy.

The outcome of interest in this study was to examine the intraindividual change in frequency of migraine attacks before, during and after ACE inhibition therapy. As a measure of change in the frequency of attacks, we used the mean consumption of abortive migraine drug use in terms of defined daily doses (DDDs) per month before, during and after ACE inhibition (index group) or diuretic treatments (reference group), as has been applied elsewhere. ${ }^{13}$ This measurement of drug utilisation was termed the 'therapeutic intensity' (TI) of abortive migraine drug use.

- 1 DDD sumatriptan corresponded to one $100 \mathrm{mg}$ tablet or one $6 \mathrm{mg}$ subcutaneous injection.

- 1 DDD naratriptan or zolmitriptan corresponded to one $2.5 \mathrm{mg}$ tablet respectively.

- 1 DDD rizatriptan corresponded to one $10 \mathrm{mg}$ tablet.

- 1 DDD ergotamine corresponded to one $4 \mathrm{mg}$ single preparation by any route or one $2 \mathrm{mg}$ combination preparation by any route. ${ }^{12}$

The TI was calculated by dividing the absolute number of DDDs dispensed per prescription per patient by the number of days between the first and last prescription presented during a 2-year follow-up period preceding, during and after ACE or diuretic therapy.

To express the impact of ACE therapy or diuretic therapy on the migraine analgesic consumption on an individual level relative to before initiation of ACE or diuretic treatments, we calculated for all patients a 'therapeutic intensity fluctuation estimate' defined as:

$$
\frac{\mathrm{TI}_{\text {during ACE or diuretic }}-\mathrm{TI}_{\text {before ACE or diuretic }}}{\mathrm{TI}_{\text {before ACE or diuretic }}} \times 100 \%
$$

In addition, the TI fluctuation estimate was calculated for those patients who had continued abortive migraine drug use even after discontinuation of ACE or diuretic treatment. This expressed the impact of discontinuing ACE or diuretic treatment on abortive

Pharmacoepidemiology and Drug Safety, 2004; 13: 41-47 
migraine drug use as compared to during the treatment with an ACE or diuretic:

$$
\frac{\mathrm{TI}_{\text {after ACE or diuretic }}-\mathrm{TI}_{\text {during ACE } \text { or diuretic }}}{\mathrm{TI}_{\text {during ACE or diuretic }}} \times 100 \%
$$

MICROSOFT ACCESS $^{\circledR}$, a relational database software package, was used for database management and internal quality and validation procedures. The difference in mean change in TI fluctuation between the index (ACE) and reference (diuretic) group was evaluated using the independent sample $t$-test as well as adjusted for differences in age, gender and prescriber using multivariate regression analysis. The statistical package SPSS for Windows was used for statistical data analysis.

\section{RESULTS}

Of the 95 included patients, $87 \%$ were women and the overall age range was $18-94$ (mean age 50.1 years). More than two-thirds included patients who were 55 years or younger. The majority of the study population (84\%) used ergotamine solely as the abortive migraine drug during the 2-year period prior to ACE or diuretic treatment (Table 1).

Recipients of the second-generation triptans were not found. Significant differences between gender and prescriber of the first $\mathrm{ACE}$ and diuretic prescription were observed when comparing the index and reference group. The ACE cohort included less females $(78.7 \%)$ than the diuretic cohort $(95.8 \%)$. The mean duration of ACE/Ang II inhibition and diuretic therapy (time span between first and last prescription) was almost identical ( $p=0.62)$; duration of treatment with ACE/Ang II inhibitors was approximately 2 years compared to 2.5 years with diuretic treatment.

For all patients, the mean TI of abortive migraine drug use before ACE/Ang II or diuretic therapy was similar, 5.5 DDDs per month and 6.7 DDDs per month respectively (Figure 2a). The intensity of abortive migraine drug use fell by $51 \%(95 \%$ CI: $36.7 \%-$ 65.3\%) for patients following ACE/Ang II treatment versus $18 \%$ (95\% CI: $7.1 \%-28.9 \%$ ) for patients using a diuretic.

For patients having continued ergotamine or sumatriptan after ACE/Ang II or diuretic therapy, similar patterns of TI were observed (Figure 2b). A reduction $(55 \%)$ followed by an increase $(36 \%)$ in the mean TI during and after discontinuation of ACE/Ang II inhibition respectively, was observed (Figure 2b). This consumption pattern of abortive migraine medication differed from that observed within the diuretic cohort, in which an increase (20\%) and a slight reduction (12\%) was observed during and after diuretic treatment.

On an individual patient level (Table 2), the change in TI 'during' relative to 'before' ACE/Ang II or diuretic therapy, expressed as the 'TI fluctuation estimate' of

Table 1. Baseline characteristics of the study population $(n=95)$

\begin{tabular}{|c|c|c|c|}
\hline Characteristics & ACE $n=47(\%)$ & Diuretic $n=48(\%)$ & $p$-value \\
\hline Gender & & & $0.01 *$ \\
\hline Female & $37(78.7)$ & $46(95.8)$ & \\
\hline Male & $10(21.3)$ & $2(4.2)$ & \\
\hline Mean (SD) Age (years) & $51.0(11.0)$ & $49.2(14.3)$ & $0.50^{\dagger}$ \\
\hline$\leq 55$ years & $31(66.0)$ & $34(70.8)$ & $0.60 *$ \\
\hline$>55$ years & $16(34.0)$ & $14(29.2)$ & \\
\hline Abortive migraine therapy before ACE or diuretic therapy & & & $0.67 *$ \\
\hline Ergotamine & $39(83.0)$ & $41(85.4)$ & \\
\hline Sumatriptan & $4(8.5)$ & $5(10.4)$ & \\
\hline Users of both & $4(8.5)$ & $2(4.2)$ & \\
\hline Abortive migraine therapy during ACE or diuretic therapy & & & $0.06^{*}$ \\
\hline Ergotamine & $39(95.1)$ & $34(79.1)$ & \\
\hline Sumatriptan & $2(4.9)$ & 5 (11.6) & \\
\hline Users of both & $0(0.0)$ & $4(9.3)$ & \\
\hline Prescriber of ACE or diuretic therapy & & & $<0.01 *$ \\
\hline General practitioner & $34(72.3)$ & 47 (97.9) & \\
\hline Specialist & $13(27.7)$ & $1(2.1)$ & \\
\hline Mean (SD) duration of ACE or diuretic therapy (years) & $2.2(2.4)$ & $2.5(2.9)$ & $0.62^{\dagger}$ \\
\hline
\end{tabular}


(a)

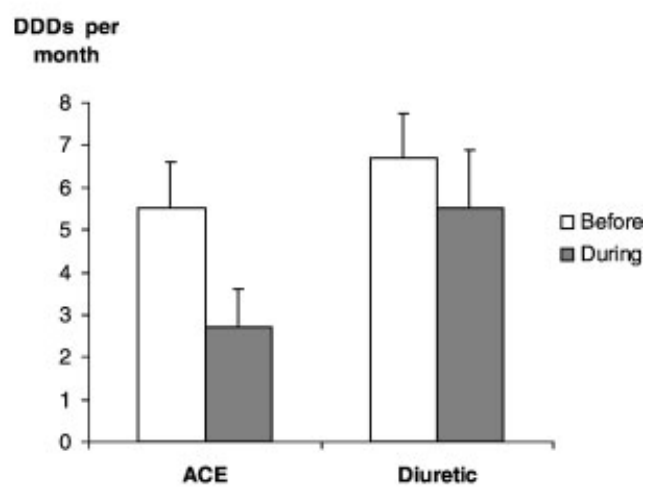

(b)

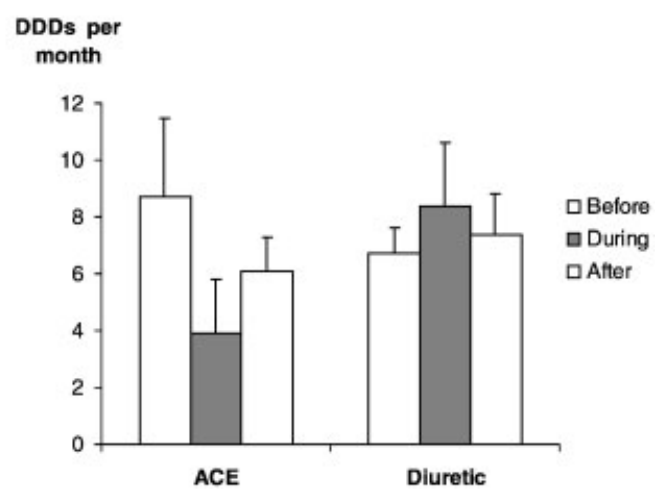

Figure 2. Therapeutic intensity of abortive migraine drug use before, during and after ACE or diuretic treatment. (a) all patients $(n=95)$ (b) Continued users $(n=47)$

abortive migraine drug use, was significantly larger for the ACE/Ang II cohort (62\% reduction) than for the diuretic group ( $24 \%$ reduction) $(p=0.02)$. This difference did not change after adjustment for age, gender and type of prescriber. Approximately, 57\% of the ACE cohort had achieved a TI reduction of $50 \%$ or more, compared to $15 \%$ of the diuretic cohort (data not shown). In contrast, an increase of $25 \%$ or more was estimated in approximately $11 \%$ of the ACE/Ang II cohort and $40 \%$ of the diuretic cohort.

For patients who continued use of abortive migraine drugs after discontinuation of ACE/Ang II $(n=20)$ or diuretic therapy $(n=27)$, a significantly larger reduction in the TI fluctuation estimate was observed in the ACE group (68.9\%) compared to the diuretic cohort $(10.5 \%$ increase $)(p=0.004)$. Following the discontinuation of ACE or diuretic therapy, the TI estimate, after relative to during therapy, increased by $50.3 \%$ for the ACE cohort and reduced by $22.2 \%$ for the diuretic cohort $(p=0.1)$.

Although the number of patients was low (data not shown), on average all ACE inhibitors prescribed led to a reduction in the TI fluctuation ratio, for which the magnitude realised by each drug was not found to be significantly different from one another. The majority of the ACE cohort, 81\%, had either been using captopril, enalapril or lisinopril during the study period and more than half of these patients had achieved an individual TI reduction in abortive migraine drug use of at least 50\% during the use of these ACE inhibitors. Only one angiotensin II receptor antagonist was included in the study, losartan, which also caused a reduction in the TI ratio in all three users of the drug.

\section{DISCUSSION}

In our study, we observed that treatment with ACE inhibitors and losartan, an ANG II, resulted in a reduction in the TI of abortive migraine drug use on an individual patient level. Close to two-thirds of our migraine patients, having undergone treatment with these drugs, had experienced a substantial reduction in abortive migraine drug use, indirectly suggesting that these patients may have experienced a reduction in the number of migraine attacks and/or attacks of shorter duration. The increase in the TI observed after discontinuing ACE inhibitors for patients continuing

Table 2. Therapeutic intensity fluctuation estimate

\begin{tabular}{|c|c|c|c|c|c|}
\hline Abortive migraine usage patterns & ACE & Diuretic & Diff & Diff adj* & $p$-value ${ }^{\dagger}$ \\
\hline All patients $(n=95)$ & $n=47$ & $n=48$ & & & \\
\hline During versus Before $(95 \% \mathrm{CI})$ & $\begin{array}{c}-62.1 \% \\
(-74.5--50.0)\end{array}$ & $\begin{array}{c}-24.0 \% \\
(-45.6-+2.4)\end{array}$ & $\begin{array}{c}38.1 \% \\
(12.2-64.0)\end{array}$ & $\begin{array}{c}34.7 \% \\
(6.2-63.2)\end{array}$ & 0.02 \\
\hline Continued users $(n=47)$ & $n=20$ & $n=27$ & & & \\
\hline During versus Before $(95 \% \mathrm{CI})$ & $\begin{array}{c}-68.9 \% \\
(-88.5--47.5)\end{array}$ & $\begin{array}{c}+10.5 \% \\
(-17.0-+38.7)\end{array}$ & $\begin{array}{c}79.4 \% \\
(35.8-122.3)\end{array}$ & $\begin{array}{c}74.8 \% \\
(24.8-124.9)\end{array}$ & 0.04 \\
\hline After versus During $(95 \% \mathrm{CI})$ & $\begin{array}{c}+50.3 \% \\
(-7.9 \%-+108.6)\end{array}$ & $\begin{array}{c}-22.2 \% \\
(-61.3-+17.1)\end{array}$ & $\begin{array}{c}72.4 \% \\
(6.1-139.9)\end{array}$ & $\begin{array}{c}61.6 \% \\
(13.6-136.9)\end{array}$ & 0.1 \\
\hline
\end{tabular}

*Adjusted for age, gender and prescriber.

${ }^{\dagger}$ Independant sample $t$-test. 
abortive migraine drugs during and after this treatment further strengthens this implication.

By comparing the efficacy of lisinopril to placebo in the prophylaxis of migraine, Schrader et al. observed a significant reduction in the number of days and duration of migraine attacks in lisinopril-treated patients. As a consequence, a reduction in the consumption of specific and non-specific migraine analgesics during lisinopril treatment was realised. ${ }^{8}$ Similar to this study, Bender et al. observed a dramatic improvement of migraine in 10 of 17 patients in whom enalapril or lisinopril was initiated. ${ }^{14}$ However, as seen in these studies as well as ours, not all patients will achieve beneficial effects from treatment with ACE inhibitors.

A possible explanation for the positive effects on migraine is the ability of the ACE inhibitors to modify sympathetic activity on vasomotor tone, decrease free radicals and increase prostacyclin production. ${ }^{15,16}$ A few genetic studies have shown that migraineurs without aura possess increased plasma ACE activity due to higher levels of an $A C E$ gene (the $A C E-D D$ gene). This association is thought to contribute to an increase in the frequency of migraine attacks in migraine without aura. ${ }^{17,18}$ The reduction in the intensity of ergotamine and sumatriptan use observed during treatment with all ACE inhibitors studied may therefore be related to this common pharmacology, assuming that amelioration of migraine was the prime cause for the reduction. Even though the use of losartan in our study did cause a reduction in the consumption of ergotamine and sumatriptan in all the three patients using this drug, we cannot fully claim that angiotensin II antagonists may offer beneficial effects in migraine prophylaxis in some patients as previously suggested, due to the small number of patients involved and lack of inclusion of other drugs from this therapeutic group. As concluded in other studies, the mechanism of this benefit is not known and randomised trials are warranted.

A few limitations of this study should not be ignored. Our study consisted of a relatively small sample size $(n=95)$, mainly due to the strict eligibility criteria applied. Hence, our results should be viewed with some caution. However, we do feel that our findings as well as others should at least stimulate interest to conduct more clinical research to further establish the effects of ACE inhibitors, as well as ACE II receptor antagonists, in migraine compared to placebo or established migraine prophylactic drugs, such as the $\beta$-blockers. We are intending to apply the same method using established migraine prophylactic drugs as the reference category in order to better validate the possible therapeutic benefit of the ACE inhibitors.

Copyright (C) 2003 John Wiley \& Sons, Ltd.
Furthermore, we estimated changes in migraine frequency and severity by analysing prescription data with the assumption that a prescription presented at the pharmacy correlates with consumption of the drug. Estimation of drug consumption is often valid for recipients of multiple prescriptions, since consistently repeated prescriptions can serve as strong evidence of drug use by patients. ${ }^{19}$ Our study included only those patients who presented multiple prescriptions of ergotamine or sumatriptan prior to ACE inhibition or diuretic treatments.

Due to the aforementioned issues as well as incomplete data concerning the use of other analgesics, particularly OTC analgesics, our estimate of migraine attack frequency on the basis of drug consumption data will have a certain degree of imprecision. However, it is highly unlikely that this imprecision is differential between the ACE and diuretic group and, therefore, the large difference found between these two groups can be justified on therapeutic grounds. In fact, various drug utilisation studies have shown that changes in drug use can be considered a reliable marker of changes in disease severity. ${ }^{20,21}$

Since access to diagnostic information was lacking, we could not distinguish between patients suffering from migraine or cluster headache. This presents as an important limitation since a positive association between cluster headache and hypertension has been demonstrated. ${ }^{22}$ This could mean that a proportion of sumatriptan users treated with an ACE inhibitor or ACE II receptor antagonist may have included patients suffering from cluster headache and co-existing hypertension. Moreover, patients suffering from cluster headache tend to require large amounts of analgesia to treat their headaches, which could alter our estimations more easily than seen for the typical migraine sufferer. However, we believe that the effect of this limitation on our estimations is minor since the majority of the population was using ergotamine and all sumatriptan users $(n=8)$ were solely using the tablet form during the observation period. Furthermore, epidemiological data reveal that the lifetime prevalence of cluster headache as compared to migraine is extremely low $(0.07 \%)$ and that an association between migraine and hypertension has likewise been established. ${ }^{21-23}$

About a third of our study population included elderly migraine patients. As a result, it could be argued that the reduction in migraine analgesic consumption in these patients may be related to the age-related decline in migraine attacks. In view of the comparable age features within the ACE and diuretic groups, a comparable reduction in migraine analgesic

Pharmacoepidemiology and Drug Safety, 2004; 13: 41-47 
consumption in the diuretic group would likely have been expected if age was a major 'contributive factor'. $7,8,20$

In view of the vasoconstrictive properties of ergotamine and sumatriptan, doctors may generally be more reluctant to prescribe these drugs to migraineurs suffering from cardiovascular disorders, such as hypertension or cardiac failure. ${ }^{24,25}$ A correlation between reluctant prescribing behaviour and the considerable reduction in migraine analgesic consumption during ACE inhibition treatment, in particular, cannot be ruled out. However, only six patients (13\%) temporarily discontinued ergotamine and sumatriptan use during this treatment.

In conclusion, we can suggest that our results indirectly contribute to the existing evidence that ACE inhibitors in particular may offer beneficial prophylactic effects to migraineurs and that these effects may be attributed to the pharmacological group as a whole. In future, these drugs could present as an alternative therapeutic option in migraineurs with coexisting cardiovascular disease, such as hypertension, particularly when $\beta$-blockers are contraindicated or are ineffective. To gain more conclusive power concerning the possible new therapeutic benefit of these drugs, more controlled clinical trials are certainly required.

\section{REFERENCES}

1. Lipton RB, Stewart WF, von Korff M. Burden of migraine: societal costs and therapeutic opportunities. Neurology 1997; 48(Suppl. 3): S4-S9.

2. Limmroth V, Michel MC. The prevention of migraine: a critical review with special emphasis on $\beta$-adrenoceptor blockers. Br J Clin Pharm 2001; 52: 237-243.

3. d' Amato CC, Pizza V, Marmolo T, et al. Fluoxetine for migraine prophylaxis: a double-blind trial. Headache 1999; 39: 716-719.

4. Schoenen J, Jacquy J, Lenaerts M. Effectiveness of high-dose riboflavin in migraine prophylaxis: a randomised controlled trial. Neurology 1998: 50: 466-470.

5. Mauskop A, Altura BM. Role of magnesium in the pathogenesis and treatment of migraines. Clin Neurosci 1998: 5: 24-27.

6. Morales-Asin F, Iniguez C, Cornudella R, et al. Patients with acenocoumarol treatment and migraine. Headache 2000; 40: $45-47$.

7. Schrader H, Stovner LJ, Helde G, et al. Prophylactic treatment of migraine with angiotensin converting enzyme inhibitor (lisinopril): randomised, placebo controlled, crossover study. Br Med J 2001; 322: 1-5.
8. Luscher TF. Angiotensin, ACE-inhibitors and endothelial control of vasomotor tone. Basic Res Cardiol 1993; 88(Suppl. 1): $15-24$.

9. Montgomery L. Are angiotensin-converting enzyme (ACE) inhibitors effective in preventing migraine in nonhypertensive patients? J Fam Pract 2001; 50: 1-2.

10. Etminan M, Tomlinson G, Levine MA, Rochon PA. Efficacy of Angiotensin II receptor antagonists in preventing headache: systematic overview and meta-regression analysis. Pharmacoepidemiol Drug Safe 2001; 10(Suppl. 1): S1.

11. Herings RMC. PHARMO; a record linkage system for postmarketting surveillance of precription drugs in The Netherlands [Dissertation]. Utrecht University 1993.

12. Anonymous. Anatomical Therapeutic Chemical (ATC) classification index. WHO Collaborating Centre for Drug Statistics Methodology: Oslo, 1993.

13. Gaist D, Halls J, Sindrup SH, Gram LF. Is overuse of sumatriptan a problem? A population based study. Eur J Clin Pharmacol 1996; 50: 161-165.

14. Bender WI. ACE inhibitors for prophylaxis of migraine headaches. Headache 1995; 35: 470-471.

15. Fabiani ME, Johnston CI. Spectrum of use for the angiotensinreceptor blocking drugs. Cur Hypertens Rep 1999; 1: 394401.

16. Martelletti $\mathrm{P}$, Cugini $\mathrm{P}$, Letizia $\mathrm{C}$, et al. Chronopathology for angiotensin converting enzyme circadian rhythm in migraine. Chronobiologia 1990; 17: 59-64.

17. Paterna S, Di Pasquale P, D'Angelo A, et al. Angiotensinconverting enzyme gene deletion polymorphism determines an increase in frequency of migraine attacks in patients suffering from migraine without aura. Eur Neurol 2000; 43: 133-136.

18. Petri H, de Vet HCW, Naus J, Urquhart J. Prescription sequence analysis: a new and fast method for assessing certain adverse reactions of prescription drugs in large populations. Stat Med 1988; 7: 1171-1175.

19. Rahimtoola H, Egberts ACG, Buurma H, Tijssen CC, Leufkens HG. Reduction in the intensity of abortive migraine drug use during coumarin therapy. Headache 2001; 41: $768-773$.

20. Hallas J, Nissen A. Individualised drug utilisation statistics: analysing a population's drug use from the perspective of individual users. Eur J Clin Pharmacol 1994; 47: 367-372.

21. Rasmussen BK, Jensen R, Schroll M, Olesen J. Epidemiology of headache in a general population-a prevalence study. J Clin Epidemiol 1991; 44: 1147-1157.

22. D'Allessandro R, Gamberini G, Benassi G, Morganti G, Cortelli P, Lugaressi E. Cluster headache in the Republic of San Marino. Cephalagia 1986; 6: 159-162.

23. Mathew NT. Migraine and hypertension. Cephalalgia 1999; 19(Suppl. 25): S17-S19.

24. Van den Brink A, Reekers M, Bax WA, Ferrari MD, Saxena PR. Coronary side-effect potential of current and prospective antimigraine drugs. Circulation 1998; 98: 25-30.

25. Young WB, Mannix L, Adelman U, Aaron L, Schecter A. Cardiac risk factors and the use of triptans: a survey study. Headache 2000; 40: 587-591. 